\title{
Ice condensation on sulfuric acid tetrahydrate: Implications for polar stratospheric ice clouds
}

\author{
T. J. Fortin ${ }^{1}{ }^{*}$, K. Drdla ${ }^{2}$, L. T. Iraci ${ }^{2}$, and M. A. Tolbert ${ }^{1}$ \\ ${ }^{1}$ Department of Chemistry and Biochemistry and Cooperative Institute for Research in Environmental Sciences, University of \\ Colorado, CIRES Building Room 318, Boulder, CO 80309-0216, USA \\ ${ }^{2}$ NASA Ames Research Center, Earth Science Division, Moffett Field, CA 94035, USA \\ *Present address: Center for Science and Technology Policy Research, University of Colorado/CIRES, 1333 Grandview \\ Avenue, Boulder, CO 80309-0488, USA
}

Received: 17 December 2002 - Published in Atmos. Chem. Phys. Discuss.: 17 February 2003

Revised: 16 May 2003 - Accepted: 3 July 2003 - Published: 9 July 2003

\begin{abstract}
The mechanism of ice nucleation to form Type 2 PSCs is important for controlling the ice particle size and hence the possible dehydration in the polar winter stratosphere. This paper probes heterogeneous ice nucleation on sulfuric acid tetrahydrate (SAT). Laboratory experiments were performed using a thin-film, high-vacuum apparatus in which the condensed phase is monitored via Fourier transform infrared spectroscopy and water pressure is monitored with the combination of an MKS baratron and an ionization gauge. Results show that SAT is an efficient ice nucleus with a critical ice saturation ratio of $\mathrm{S}_{\text {ice }}^{*}=1.3$ to 1.02 over the temperature range $169.8-194.5 \mathrm{~K}$. This corresponds to a necessary supercooling of $0.1-1.3 \mathrm{~K}$ below the ice frost point. The laboratory data is used as input for a microphysi$\mathrm{cal} /$ photochemical model to probe the effect that this heterogeneous nucleation mechanism could have on Type 2 PSC formation and stratospheric dehydration. In the model simulations, even a very small number of SAT particles (e.g., $10^{-3} \mathrm{~cm}^{-3}$ ) result in ice nucleation on SAT as the dominant mechanism for Type 2 PSC formation. As a result, Type 2 PSC formation is more widespread, leading to larger-scale dehydration. The characteristics of the clouds are controlled by the assumed number of SAT particles present, demonstrating that a proper treatment of SAT is critical for correctly modeling Type 2 PSC formation and stratospheric dehydration.
\end{abstract}

\section{Introduction}

Polar stratospheric clouds (PSCs) have been observed for over a century (Stanford and Davis, 1974). However, it was not until the latter half of the twentieth century that their relevance to atmospheric chemistry was realized (Crutzen and

Correspondence to: T. J. Fortin

(fortint@ cires.colorado.edu)
Arnold, 1986; Solomon et al., 1986). For example, PSCs serve as surfaces upon which heterogeneous reactions occur that convert nonreactive halogen species to photochemically reactive forms that participate in the catalytic destruction of ozone (McElroy et al., 1986; Molina, 1991). In addition, PSCs play a pivotal role in the redistribution of chemical species throughout the stratosphere. Specifically, the sedimentation of large PSC particles can result in the irreversible removal of $\mathrm{HNO}_{3}$. This process of denitrification can enhance ozone loss by delaying the sequestration of ozone-destroying chlorine species (Toon et al., 1986). Moreover, the sedimentation of PSCs can result in dehydration by removing $\mathrm{H}_{2} \mathrm{O}$, with possible consequences for subsequent PSC formation, the hydrogen oxide $\left(\mathrm{HO}_{\mathrm{x}}\right)$ budget, and the stratosphere's radiation balance (Mancini et al., 1992; Schiller et al., 1996). Finally, as the PSCs fall, they can release these chemical species back into the atmosphere at lower altitudes.

Polar stratospheric clouds are commonly divided into two classes based on their formation temperature and composition (Browell et al., 1990; Turco et al., 1989). Type 1 PSCs contain $\mathrm{HNO}_{3}$ and form at approximately 190 to $195 \mathrm{~K}$. Type 1 clouds are further separated into at least two subdivisions depending on whether the $\mathrm{HNO}_{3}$ is in crystalline form (Type 1a), presumably as nitric acid trihydrate (NAT) or nitric acid dihydrate (NAD), or in solution as ternary $\mathrm{HNO}_{3} / \mathrm{H}_{2} \mathrm{O} / \mathrm{H}_{2} \mathrm{SO}_{4}$ (Type 1b). The second type of PSC (Type 2) is composed of water ice and forms at temperatures near the ice frost point $(\sim 188-190 \mathrm{~K})$. This paper focuses on a possible formation mechanism for Type 2 PSCs.

There are currently several theories on how Type 2 PSCs are formed: (1) vapor deposition of ice on top of NAT; (2) homogeneous nucleation of ice out of $\mathrm{H}_{2} \mathrm{SO}_{4} / \mathrm{H}_{2} \mathrm{O}$, $\mathrm{H}_{2} \mathrm{SO}_{4} / \mathrm{HNO}_{3} / \mathrm{H}_{2} \mathrm{O}$, or $\mathrm{HNO}_{3} / \mathrm{H}_{2} \mathrm{O}$ liquid aerosol; and (3) heterogeneous nucleation of ice out of liquid aerosol containing insoluble nuclei such as mineral oxides or soot. Of these theories, the second is perhaps the most commonly invoked.

(C) European Geosciences Union 2003 
However, homogeneous nucleation of ice out of solutions such as $\mathrm{H}_{2} \mathrm{SO}_{4} / \mathrm{H}_{2} \mathrm{O}, \mathrm{HNO}_{3} / \mathrm{H}_{2} \mathrm{O}$, or $\mathrm{H}_{2} \mathrm{SO}_{4} / \mathrm{HNO}_{3} / \mathrm{H}_{2} \mathrm{O}$ requires supercooling on the order of $2-3 \mathrm{~K}$ below the ice frost point (Bertram et al., 1996; Chang et al., 1999; Jensen et al., 1991; Koop et al., 1998; Middlebrook et al., 1993; Tabazadeh et al., 1997a, 1997b). In contrast, theoretical work suggests that the third nucleation mechanism, heterogeneous nucleation in aqueous solutions containing solid cores, may occur at temperatures warmer than those required for homogeneous nucleation (DeMott et al., 1997; Jensen and Toon, 1997; Sassen and Benson, 2000). However, there is limited laboratory data available to support these claims (Chen et al., 2000; Zuberi et al., 2001), all of which has focused on conditions and heterogeneous nuclei that are relevant to the troposphere. Finally, while no studies could be found that reported the vapor deposition of ice on NAT, Barone et al. (1997) observed that the nucleation of ice on NAT surfaces having a thin aqueous nitric acid layer on top requires a supercooling of $\sim 2 \mathrm{~K}$ below the ice frost point.

In this paper, we report on the possibility of a fourth nucleation mechanism for Type 2 PSCs, vapor deposition of ice on top of sulfuric acid tetrahydrate (SAT). While there is field (David et al., 1998; Dye et al., 1992), laboratory (Anthony et al., 1995; Carleton et al., 1997; Koop et al., 1997b), and theoretical (Luo et al., 1994) evidence that background sulfate aerosols often remain liquid down to very low temperatures, this is not always the case. Results from both the laboratory (Iraci et al., 1995; Koop et al., 1995; Middlebrook et al., 1993; Molina et al., 1993) and the field (Beyerle et al., 2001; Gobbi and Adriani, 1993; Larsen et al., 1995; Nagai et al., 1997; Rivière et al., 2000; Rosen et al., 1993; Sassen et al., 1994) suggest that sulfate aerosols could, even if only in small numbers, exist in a frozen state. According to the bulk thermodynamics (Gable et al., 1950; Koop et al., 1997a), SAT is the stable phase of such aerosols under most stratospheric conditions. It is important to note that, in contrast to stratospheric conditions, the laboratory experiments reported here have been performed in the absence of $\mathrm{HNO}_{3}$ vapor. This is relevant since previous work has shown that the presence of $\mathrm{HNO}_{3}$ renders SAT thermodynamically unstable at temperatures below the nitric acid "dew point" (Koop and Carslaw, 1996). However, the results of Iraci et al. (1998) indicate that the kinetics of this dissolution process may limit its occurrence in the atmosphere. This is demonstrated by the fact that only approximately $2 / 3$ of the SAT samples tested in that study exhibited a phase transformation forming ternary $\mathrm{HNO}_{3} / \mathrm{H}_{2} \mathrm{SO}_{4} / \mathrm{H}_{2} \mathrm{O}$ solution. Furthermore, formation of NAT would preclude the dissolution of SAT (Koop et al., 1997a), thereby highlighting the possibility that the growth of Type 2 PSCs on polycrystalline NAT/SAT particles may occur on either of these surfaces.

To address whether or not SAT could act as an effective ice nucleus, we have performed isothermal ice nucleation experiments on SAT films. In these studies, the saturation ratio with respect to ice is increased incrementally while we

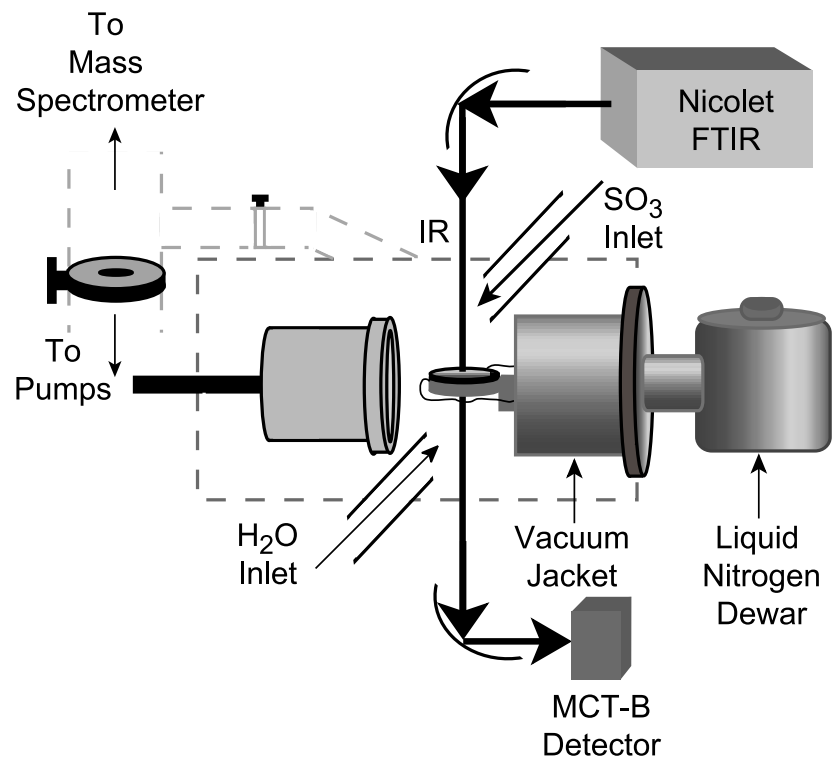

Fig. 1. Schematic diagram of the experimental apparatus.

probe for ice nucleation using transmission Fourier transform infrared (FTIR) spectroscopy. The results of these laboratory experiments have been incorporated into a microphysical model to assess whether ice nucleation on SAT could play an important role in the stratosphere. Specifically, simulations with a variety of assumed SAT particle concentrations have been performed to test the effect of this nucleation mechanism on Type 2 PSC existence temperatures, characteristics (e.g. ice particle radii and number concentration), and stratospheric dehydration.

\section{Experimental}

\subsection{Thin-film apparatus}

Experiments were performed using a thin-film, high-vacuum apparatus similar to that previously described in Fortin et al. (2002) and shown schematically in Fig. 1. Briefly, a silicon wafer $(2.5 \mathrm{~cm}$ diameter, $1 \mathrm{~mm}$ thick) is supported on a copper mount that is in thermal contact with a liquid nitrogen reservoir. A stainless steel vacuum jacket isolates the cold finger from the remainder of the chamber; thereby ensuring the mount is the only exposed cold surface. Temperature is controlled by heating an annular resistive Kapton heater located between the copper mount and the cold finger via use of a Eurotherm temperature controller. The temperature is measured using T-type thermocouples that are attached to the copper mount at two different locations and are monitored by a PC utilizing LabView data collection software. The thermocouples are calibrated at the end of each experiment via reference to the ice frost point (Marti and Mauersberger, 1993). The $\mathrm{H}_{2} \mathrm{O}$ pressure is adjusted until 


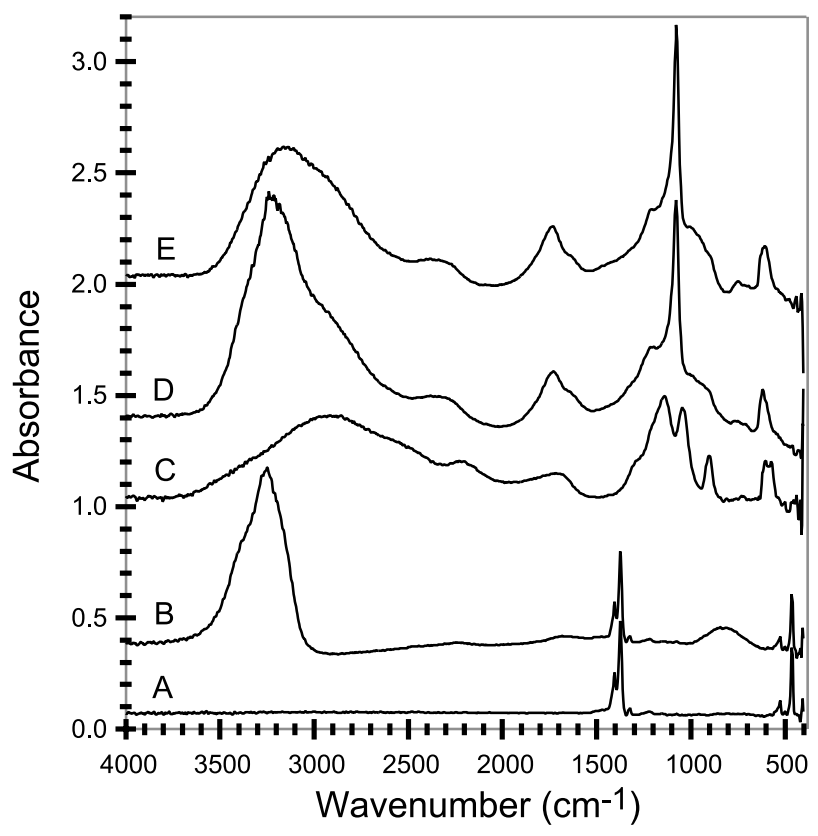

Fig. 2. Making a SAT film. $\mathrm{SO}_{3}$ is vapor deposited at $110 \mathrm{~K}$ (spectrum A). Ice is then deposited on top of the $\mathrm{SO}_{3}$ (spectrum $\mathrm{B}$ ). Warming the film at $5 \mathrm{~K} \mathrm{~min}^{-1}$ to $215 \mathrm{~K}$ forms a liquid $\mathrm{H}_{2} \mathrm{SO}_{4}$ film (spectrum C). Cooling in the presence of water vapor to $170 \mathrm{~K}$ crystallizes both ice and SAT (spectrum D). Finally, warming the film evaporates the ice, leaving behind a SAT film (spectrum E).

the integrated area of the ice infrared libration peak at 779$879 \mathrm{~cm}^{-1}$ is constant, indicating that the ice film is no longer growing or evaporating. Once the necessary correction (usually less than $2 \mathrm{~K}$ ) is applied, temperatures are accurate to within either $\pm 0.2 \mathrm{~K}$ or $\pm 0.4 \mathrm{~K}$, depending on conditions. For the $\mathrm{H}_{2} \mathrm{O}$ source, a liquid $\mathrm{H}_{2} \mathrm{O}$ reservoir (HPLC Grade, Fisher Scientific) is purified at the start of each day by freezing the bulb in a liquid nitrogen bath and pumping off any gaseous impurities. The reservoir is then thawed and the vapors are introduced into the chamber through a leak valve. Condensed-phase species are monitored with a Nicolet 740 FTIR spectrometer. For each experiment, the spectral resolution is $8 \mathrm{~cm}^{-1}$ and spectra are collected approximately every five seconds. Finally, water pressure is measured by a combination of an MKS Baratron gauge and a calibrated ionization gauge, both of which are monitored with the same PC used for the thermocouples. A calibrated UTI 100C quadrupole mass spectrometer is used to verify that $\mathrm{H}_{2} \mathrm{O}$ is the only gasphase species present in the chamber, and can also be used to measure $\mathrm{H}_{2} \mathrm{O}$ pressure. The estimated error for pressure measurements ranges from $\pm 2 \%$ to $\pm 10 \%$, depending on the gauge used and the specific experimental conditions.

\subsection{Sulfuric acid tetrahydrate films}

SAT films are made in situ as illustrated in Fig. 2, which shows spectra at various points during the preparation of a film. First, $\mathrm{SO}_{3}$ vapor is deposited on top of the silicon wafer at approximately $110 \mathrm{~K}$ (spectrum A). The vapors over a reservoir of solid $\mathrm{SO}_{3}$ (Aldrich Chemical Co.) are introduced into the chamber via a separate leak valve for this purpose. Next, approximately $1-1.5 \mu \mathrm{m}$ of ice is deposited on top of the $\mathrm{SO}_{3}$ (spectrum B). The film is then warmed at $5 \mathrm{~K} \mathrm{~min}^{-1}$ to approximately $215 \mathrm{~K}$ to form a liquid sulfuric acid film (spectrum C). The $\mathrm{H}_{2} \mathrm{SO}_{4}$ film is then cooled in presence of $\mathrm{H}_{2} \mathrm{O}$ to $170 \mathrm{~K}$, at which point both ice and SAT are crystallized (spectrum D). The addition of $\mathrm{H}_{2} \mathrm{O}$ prevents the formation of sulfuric acid monohydrate (SAM). The film is then warmed in the absence of $\mathrm{H}_{2} \mathrm{O}$ to either 180,185 , or $190 \mathrm{~K}$. The ice evaporates, leaving behind a SAT film (spectrum E). The film shown in spectrum E compares very well to previously published spectra for SAT (Middlebrook et al., 1993; Nash et al., 2000; Zhang et al., 1993), with prominent peaks at $1077 \mathrm{~cm}^{-1}\left(\mathrm{SO}_{4}^{2-}\right.$ asymmetric stretch $), 1730 \mathrm{~cm}^{-1}$ $\left(\mathrm{H}_{3} \mathrm{O}^{+}\right.$asymmetric bend), and $3148 \mathrm{~cm}^{-1}$ (OH stretch). At the end of an experiment, the usual procedure is to warm the film to $215 \mathrm{~K}$ to reform a liquid $\mathrm{H}_{2} \mathrm{SO}_{4}$ film, which is then recrystallized to SAT following the above procedure. However, on five different occasions the SAT film was regenerated by merely warming to $190 \mathrm{~K}$ to drive off the condensed ice. Once the $\mathrm{H}_{2} \mathrm{O}$ had been pumped away, the SAT film could be cooled to a desired temperature in preparation for a new experiment. It is important to note that these two distinct treatments of the SAT film do not appear to affect the results. However, both procedures could result in SAT surfaces that are preactivated for ice formation, similar to what has been suggested for NAT nucleation on SAT (Zhang et al., 1996). This point will be addressed further in the following section.

\subsection{Procedure}

In each experiment, the temperature is set and allowed to stabilize. Water vapor is then introduced into the chamber. The water pressure is increased incrementally while the film is monitored with transmission FTIR spectroscopy for signs of ice. Condensed ice is indicated by absorption bands at $3220 \mathrm{~cm}^{-1}, 1690 \mathrm{~cm}^{-1}$ and $820 \mathrm{~cm}^{-1}$, which correspond to the $\mathrm{OH}$ stretch $\left(v_{s}\right)$, the $\mathrm{HOH}$ bend $\left(v_{2}\right)$, and the hindered rotation (or libration) $\left(v_{L}\right)$, respectively (Eisenberg and Kauzmann, 1969). The ice film is then allowed to grow so that an ice frost point calibration can be performed. Finally, the film is reprocessed in one of the two manners described above to prepare for another experiment.

\section{Results and discussion}

Figure 3a shows select spectra from a typical experiment conducted at $173.3 \mathrm{~K}$. Spectrum 1 is that of the SAT film at the start of the experiment prior to introducing additional $\mathrm{H}_{2} \mathrm{O}$ into the chamber. The $\mathrm{H}_{2} \mathrm{O}$ pressure $\left(P_{\mathrm{H}_{2} \mathrm{O}}\right)$ at this point was less than $1 \times 10^{-6}$ torr. Water vapor was then introduced 


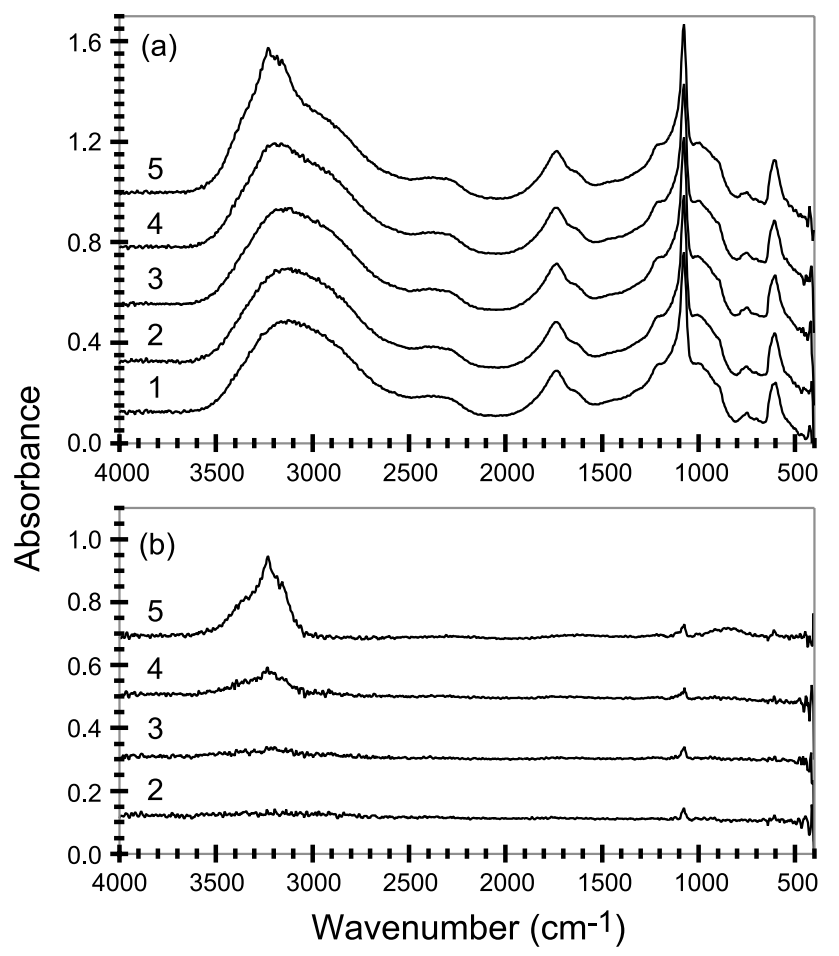

Fig. 3. Spectral results for an ice deposition experiment at $173.3 \mathrm{~K}$. The top panel (a) shows selected IR spectra. The bottom panel (b) shows the results after spectrum 1 has been subtracted out of each subsequent spectrum. In both panels, the spectra have been offset for clarity. Spectrum 1 is the initial SAT film with $P_{\mathrm{H}_{2} \mathrm{O}}<1 \times$ $10^{-6}$ torr. As $P_{\mathrm{H}_{2} \mathrm{O}}$ is increased, no ice nucleation is apparent until spectrum $4\left(P_{\mathrm{H}_{2} \mathrm{O}}=1.5 \times 10^{-5}\right.$ torr $)$. Ice features are more distinct a few minutes later (spectrum 5 ) once the ice film has had a chance to grow.

into the chamber to a pressure of $P_{\mathrm{H}_{2} \mathrm{O}}=1.2 \times 10^{-5}$ torr. After approximately nineteen minutes at this pressure, spectrum 2 was obtained, showing no signs of ice growth. The experiment continued by slowly increasing the $\mathrm{H}_{2} \mathrm{O}$ pressure, waiting at least two minutes between each pressure increase. Spectrum 3 was obtained twenty-five minutes into the experiment at $P_{\mathrm{H}_{2} \mathrm{O}}=1.4 \times 10^{-5}$ torr. Again, no ice growth is apparent. Spectrum 4 was obtained after increasing the pressure to $P_{\mathrm{H}_{2} \mathrm{O}}=1.5 \times 10^{-5}$ torr. At this point, the appearance of the $\mathrm{OH}$ stretch region begins to change, becoming sharper than in the previous spectrum, an indication of crystalline ice. Spectrum 5 was taken just four minutes later, without adjusting the $\mathrm{H}_{2} \mathrm{O}$ pressure. The $\mathrm{OH}$ stretch region has become more crystalline in appearance as the ice has continued to grow in. Ice growth is also indicated by $P_{\mathrm{H}_{2} \mathrm{O}}$, which has dropped to $1.4 \times 10^{-5}$ torr as the vapor continues to condense on the growing ice film.

While the spectra shown in Fig. 3a do show ice growth, spectral changes are more readily seen in spectral subtractions, such as those shown in Fig. 3b. Here, spectrum 1 has

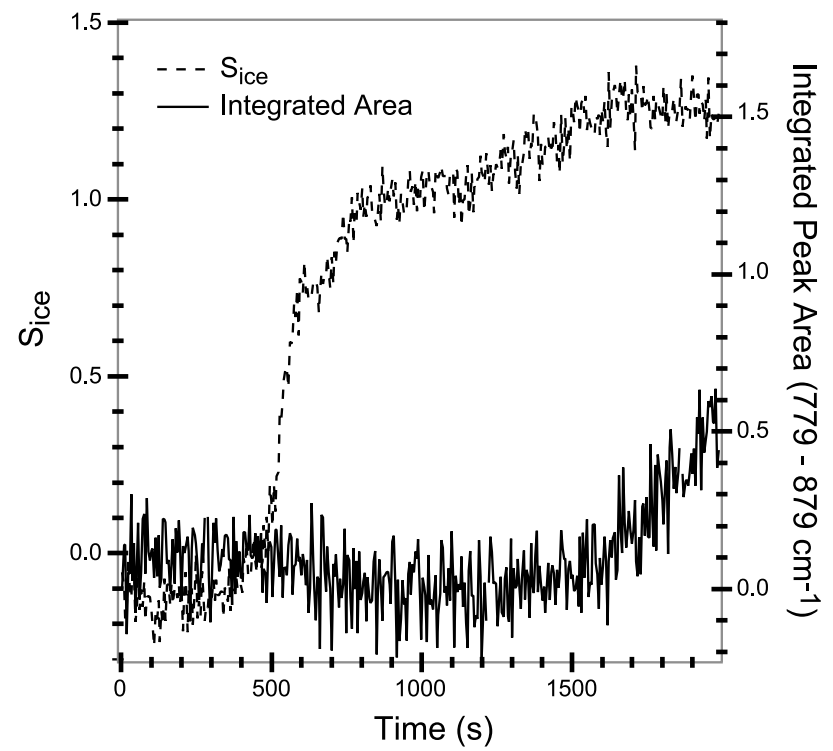

Fig. 4. Determination of the critical saturation ratio. The saturation ratio with respect to ice is plotted vs. time for the experiment shown in Fig. 3 (dashed line). Also shown is the integrated area 779 to $879 \mathrm{~cm}^{-1}$ plotted vs. time (solid line). Ice nucleation is indicated by a sharp increase in the integrated area. The corresponding saturation ratio is referred to as the critical saturation ratio, $S_{\text {ice }}^{*}$. For this experiment, $S_{\text {ice }}^{*}=1.3 \pm 0.1$.

been subtracted from each subsequent spectrum, and is therefore not shown. The remaining subtraction results are labeled accordingly. These results verify the above observations. No signs of ice growth are apparent until spectrum 4. At this point, peaks corresponding to the OH stretch $\left(3220 \mathrm{~cm}^{-1}\right)$ and the libration $\left(820 \mathrm{~cm}^{-1}\right)$ for ice are evident. These peaks become more conspicuous as the ice film continues to grow (spectrum 5).

Although both the spectra and spectral subtractions are valuable for determining the presence of ice, we do not use them to determine the onset of nucleation. Rather, we use integrated areas of two ice features previously discussed. Figure 4 shows the integrated area of the libration at 779 $879 \mathrm{~cm}^{-1}$ plotted as a function of time for the same experiment shown in Fig. 3. The integrated area of the $\mathrm{OH}$ stretch at $3181-3282 \mathrm{~cm}^{-1}$ plotted as a function of time is also used, but is not shown here. Both integration areas yield similar results. In addition to the integrated peak area, Fig. 4 also shows the saturation ratio with respect to ice $\left(S_{\text {ice }}\right)$ plotted as a function of time. $S_{\text {ice }}$ is defined as

$S_{\text {ice }}=\frac{P_{\mathrm{H}_{2} \mathrm{O}}(T)}{P_{\mathrm{eq}}(T)}$,

where $P_{\mathrm{H}_{2} \mathrm{O}}(T)$ is the experimental $\mathrm{H}_{2} \mathrm{O}$ pressure for some experimental temperature $T$ and $P_{\mathrm{eq}}(T)$ is the equilibrium vapor pressure of ice at that same temperature, as determined using the expression of Marti and Mauersberger (1993). 


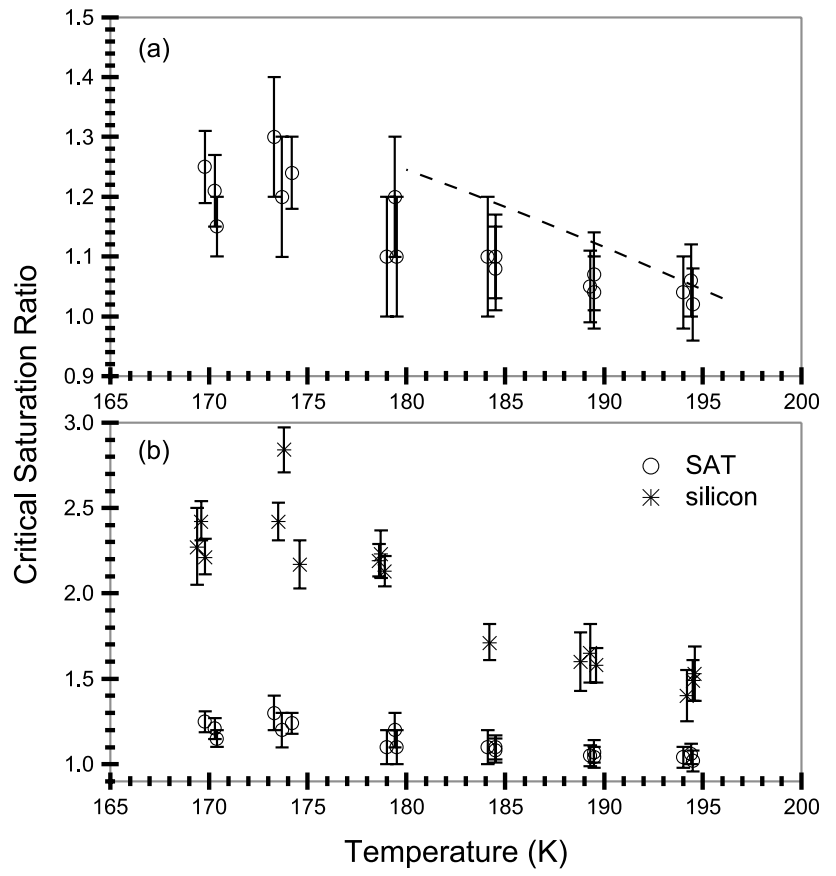

Fig. 5. Summary of experimental results. The top panel (a) shows critical saturation ratios plotted as a function of temperature for all ice on SAT experiments. The dashed line represents the ice saturation ratio at the point SAT becomes unstable, determined using the European Aerosol Inorganics Model (AIM) (Carslaw et al., 1995; Massucci et al., 1999). The bottom panel (b) compares the results from ice on SAT experiments with the results from ice on silicon experiments. In all cases, ice nucleation on silicon requires a significantly higher $S_{\text {ice }}^{*}$. Error bars are determined by propagation of measurement errors. Temperature error bars are on the order of the symbol size $( \pm 0.2-0.4 \mathrm{~K})$ and therefore are not shown.

Figure 4 shows that as $S_{\text {ice }}$ is increased, no ice nucleates until some critical saturation ratio $\left(S_{\text {ice }}^{*}\right)$ is reached. At this point, the integrated area increases dramatically, indicating ice has nucleated and is growing. It is this increase in the integrated area profile that is used to determine $S_{\text {ice }}^{*}$. For this experiment, $S_{\text {ice }}^{*}=1.3 \pm 0.1$, corresponding to a critical $\mathrm{H}_{2} \mathrm{O}$ pressure $\left(P_{\mathrm{H}_{2} \mathrm{O}}^{*}\right)$ of $1.5 \times 10^{-5}$ torr. The error in $S_{\text {ice }}^{*}$ is determined by propagating the estimated errors in both the water pressure measurement $( \pm 2 \%$ to $\pm 10 \%)$ and the temperature measurement $( \pm 0.2$ to $\pm 0.4 \mathrm{~K})$.

The results for $S_{\text {ice }}^{*}$ as a function of temperature are summarized in Fig. 5a. It can be seen in this figure that SAT appears to be an efficient ice nucleus with $S_{\text {ice }}^{*}=1.02-1.3$ over the temperature range 194.5-169.8 K. This corresponds to a necessary supercooling of only $0.1-1.3 \mathrm{~K}$ below the ice frost point. When compared to the $2-3 \mathrm{~K}$ supercooling required for homogeneous nucleation (Bertram et al., 1996; Chang et al., 1999; Jensen et al., 1991; Koop et al., 1998; Middlebrook et al., 1993; Tabazadeh et al., 1997a, 1997b), our data indicates that vapor deposition of ice on SAT could be a competitive nucleation mechanism. The second observation is that there is a slight temperature dependence in $S_{\text {ice }}^{*}$, with $S_{\text {ice }}^{*}$ increasing with decreasing temperature. The cause of this behavior is not entirely clear, although we offer one possible explanation below.

For comparison, Fig. 5b contains the same $S_{\text {ice }}^{*}$ data for ice on SAT in addition to $S_{\text {ice }}^{*}$ data for the vapor deposition of ice on silicon. Note the change in scale. These additional experiments were performed in the same manner as was previously described for ice on SAT but without a SAT film present. They were performed to verify that the previously observed ice nucleation occurred on SAT rather than on any exposed silicon or on the copper mount surfaces. It can be seen that at each temperature, $S_{\text {ice }}^{*}$ for ice on silicon is significantly higher than $S_{\text {ice }}^{*}$ for ice on SAT. Therefore, vapor deposition of ice is easier on SAT than on any other exposed surfaces that may exist in the apparatus and, as a result, will occur preferentially.

Our observation of efficient ice deposition on SAT raises two questions regarding the details of the nucleation process. First, in light of how SAT is prepared in this study, one possibility is that our SAT surfaces could be preactivated towards ice formation, thereby enhancing their efficiency as ice nuclei. However, since SAT is most likely to form in the stratosphere via a heterogeneous mechanism such as that utilized here (e.g., Peter, 1997), the distinction between SAT and preactivated SAT in this study is immaterial to the implications of the results. More importantly, despite the absence of $\mathrm{HNO}_{3}$ in these experiments, the possibility of SAT dissolution (or "deliquescence") still needs to be considered (Koop and Carslaw, 1996). The dashed line in Fig. 5a represents $S_{\text {ice }}$ at the point SAT becomes thermodynamically unstable upon cooling, determined using the European Aerosol Inorganics Model (AIM) (Carslaw et al., 1995; Massucci et al., 1999). Comparison of this line to the experimental data demonstrates that, particularly at the higher temperatures, SAT dissolution followed by heterogeneous ice nucleation within the resulting liquid layer is indeed a possible mechanism. While neither the infrared spectra nor the spectral subtractions ever showed the formation of aqueous $\mathrm{H}_{2} \mathrm{SO}_{4}$ (see Fig. 3), the presence of a small amount of liquid below our detection limit cannot be ruled out. Integrating the SAT peak from 1060 to $1095 \mathrm{~cm}^{-1}$ showed a possible decrease in the peak area suggestive of a few percent decrease in the amount of SAT present in a fraction of our experiments. However, the magnitude of the decrease in signal is comparable to the spectral noise rendering this analysis inconclusive. Therefore, based on the available spectroscopic evidence, we believe we are observing the vapor deposition of ice on SAT.

Using our experimentally determined $S_{\text {ice }}^{*}$ data, it is possible to calculate a contact parameter, $m$, between ice and SAT for vapor deposition; keeping in mind that the following calculations would be somewhat altered if the mechanism is actually that of heterogeneous nucleation out of solution. The contact parameter is a measure of the degree of matching 
between these two lattices. A perfect match is indicated by $m=1$, while a complete mismatch is indicated by $m=-1$. To calculate $m$, we use classical heterogeneous nucleation theory for nucleation on a planar substrate. Pruppacher and Klett (1997) define the nucleation rate, $J$, as

$J=A \mathrm{e}^{\left(-\frac{\Delta F}{k T}\right)}$,

where $A$ is the prefactor, $k$ is the Boltzmann constant, $T$ is the temperature in Kelvin, and $\Delta F$ is the free energy associated with critical-sized germ formation. For ice nucleation, Fletcher (1962) estimated an $A$ on the order of $10^{24}$ to $10^{27} \mathrm{~cm}^{-2} \mathrm{~s}^{-1}$. Following this example, we use the approximations $J=1 \mathrm{~cm}^{-2} \mathrm{~s}^{-1}$ and $A=10^{25} \mathrm{~cm}^{-2} \mathrm{~s}^{-1}$ in our calculations. Such approximations are justified by the fact that $m$ is not very sensitive to either of these terms. For example, varying $J$ or $A$ by twenty-five orders of magnitude changes $m$ by $\sim 2 \%$. By rearranging Eq. (2) we can calculate $\Delta F$, which is related to the experimentally determined $S_{\text {ice }}^{*}$ by the following expression

$\Delta F=\frac{16 \pi M^{2} \sigma_{i v}^{3}}{3\left[R T \rho \ln S_{\text {ice }}^{*}\right]^{2}} f(m)$,

where $f(m)$ is a matching function, $R$ is the universal gas constant, and $M$ is the molecular weight $\left(18.0152 \mathrm{~g} \mathrm{~mol}^{-1}\right)$. The density of ice, $\rho$, was calculated as a function of temperature using the expression of Pruppacher and Klett (1997)

$\rho=\sum_{n=0}^{2} a_{n} T\left({ }^{\circ} \mathrm{C}\right)^{n}$,

with $a_{0}=0.9167, a_{1}=-1.75 \times 10^{-4}$, and $a_{2}=-5.0 \times$ $10^{-7}$. Finally, $\sigma_{i v}$ is the surface tension at the ice/vapor interface. The surface tension for both the ice/water and water/vapor interfaces exhibits a temperature dependence, the expressions for which are available in the literature (Pruppacher and Klett, 1997). Unfortunately, we were unable to find an explicit expression in the literature for the temperature dependence of $\sigma_{i v}$. However, Pruppacher and Klett (1997) cite values of $\sigma_{i v}=104 \mathrm{erg} \mathrm{cm}^{-2}$ at $273 \mathrm{~K}$ and $\sigma_{i v}=106 \mathrm{erg} \mathrm{cm}^{-2}$ at $233 \mathrm{~K}$. To estimate the temperature dependence of $\sigma_{i v}$, we fit these two points with a straight line. Using the resulting expression

$\sigma_{i v}=-5 \times 10^{-2} * T\left({ }^{\circ} \mathrm{C}\right)+1.04 \times 10^{2}$,

we were able to calculate $\sigma_{i v}$ at each of our experimental temperatures. Our resultant $\sigma_{i v}$ values range from 109.2 to $107.9 \mathrm{erg} \mathrm{cm}^{-2}$ over the temperature range $169.8-194.5 \mathrm{~K}$. By comparison, over the same temperature range, estimates of the temperature dependence for the ice/liquid water interface yields values of 112-116 $\mathrm{erg} \mathrm{cm}^{-2}$ (Drdla and Turco, 1991). Therefore, our estimate of the temperature dependence of $\sigma_{i v}$ does not seem unreasonable. By rearranging Eq. (3) and solving for $f(m), m$ can be calculated using

$f(m)=\frac{(2+m)(1-m)^{2}}{4}$.

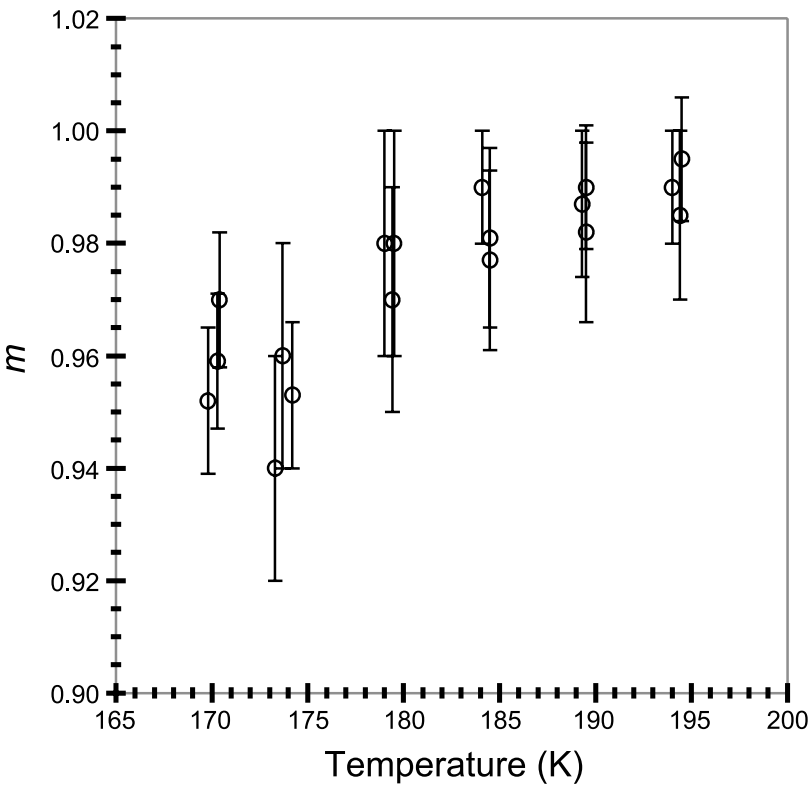

Fig. 6. Contact parameter results. The calculated contact parameter between ice and SAT is plotted as a function of temperature for all experiments in this study. Error bars are determined by propagation of errors. Temperature error bars are on the order of the symbol size $( \pm 0.2-0.4 \mathrm{~K})$ and therefore are not shown.

Solving Eq. (6) numerically results in a determination of $m$. The results of these calculations are summarized in Fig. 6.

Figure 6 shows that $m$ ranges from 0.94 to 0.995 over the temperature range $169.8-194.5 \mathrm{~K}$. This temperature dependence in $m$ is surprising. If $m$ truly represents the lattice matching between SAT and ice, one would expect a single value for $m$. One possible explanation for the observed temperature dependence could be that the crystal structures change over our temperature range. At our warmest temperatures we are confident that we are vapor depositing hexagonal ice. In contrast, at the colder temperatures we may be vapor depositing some fraction of the ice as cubic ice. The literature lends some credence to this hypothesis. There is a significant body of experimental evidence that puts the transition temperature between vapor deposition of hexagonal ice and vapor deposition of cubic ice at anywhere between 193 and $143 \mathrm{~K}$ (Hobbs, 1974).

Since the temperature dependence in $m$ mirrors that observed in $S_{\text {ice }}^{*}$ (Fig. 5), an alternative explanation for the observed temperature dependence is that the behavior in $m$ is an artifact of the calculation. If we had neglected the temperature dependence of either $\sigma_{i v}$ or $\rho$, this might be the case. However, since we have accounted for these dependencies in our calculations, we believe that the observed behavior is real, and thus favor the former explanation. Regardless of the cause of the temperature dependence, the results reported here indicate that SAT is an efficient ice nucleus. 


\section{Atmospheric implications}

Since SAT is an effective ice nucleus, the vapor deposition of ice on SAT could compete with, and perhaps even dominate, the ice nucleation process in the formation of Type 2 PSCs. This, in turn, could significantly change our understanding of the existence temperature and properties of these clouds. To investigate this further, we have employed a microphysical/photochemical model to simulate Type 2 PSC formation when the vapor deposition of ice on SAT is invoked.

The model employed, the Integrated MicroPhysics and Aerosol Chemistry on Trajectories (IMPACT) model, is discussed in detail elsewhere (Drdla, 1996; Drdla et al., 2003). Briefly, the IMPACT model is a Lagrangian trajectory model that traces an individual air parcel as it moves through the atmosphere. It simulates the PSC microphysics (condensation, evaporation, sedimentation, nucleation, freezing, and melting), heterogeneous chemistry, and gas-phase chemistry. The model sedimentation scheme includes a calculation of the incoming particle flux from higher altitudes (causing renitrification and rehydration) as discussed by Drdla et al. (2003).

The default mechanism for water ice formation in the model is homogeneous freezing of sulfate aerosol, using the rates of Tabazadeh et al. (2000). Heterogeneous nucleation can also be simulated. To include the heterogeneous nucleation of ice on SAT in the model, the data in Fig. 6 was fit with the following expression

$m(T)=-0.468262+(0.0144292 * T)+$

$\left(-3.55968 \times 10^{-5} * T^{2}\right)$

where $T$ is the temperature in Kelvin. Equation (7) is valid over the range $170<T<195 \mathrm{~K}$. A prescribed number of SAT particles is assumed to be initially present, ranging from $1 \mathrm{~cm}^{-3}$ to $10^{-5} \mathrm{~cm}^{-3}$ in different simulations. SAT is assumed to form by freezing at temperatures warmer than the ice frost point and to melt when temperatures exceed the SAT melting point (Zhang et al., 1993). The remainder of the aerosol remains liquid unless homogeneous freezing (Tabazadeh et al., 2000) is able to occur. Given the focus on Type 2 PSCs, no other PSC formation mechanisms were included in these simulations.

The model runs examined Type 2 PSC formation and dehydration during the 1999-2000 Arctic winter. Given the warmer temperatures in the Arctic than in the Antarctic, Arctic Type 2 PSC formation is more difficult and therefore more likely to be sensitive to the assumed formation mechanisms. The winter of 1999-2000 was particularly cold in the Arctic and a maximum dehydration of 0.63 ppmv was observed (Herman et al., 2003; Schiller et al., 2002). As in Drdla et al. (2003), a set of 2905 diabatic trajectories was used to provide complete coverage of the vortex for the entire winter. The trajectories were calculated from the standard $3.75 \mathrm{deg}$ $\times 2.5 \mathrm{deg}$ UKMO $12 \mathrm{Z}$ analyses, and therefore only resolve synoptic scale cooling. One limitation of these trajectories is

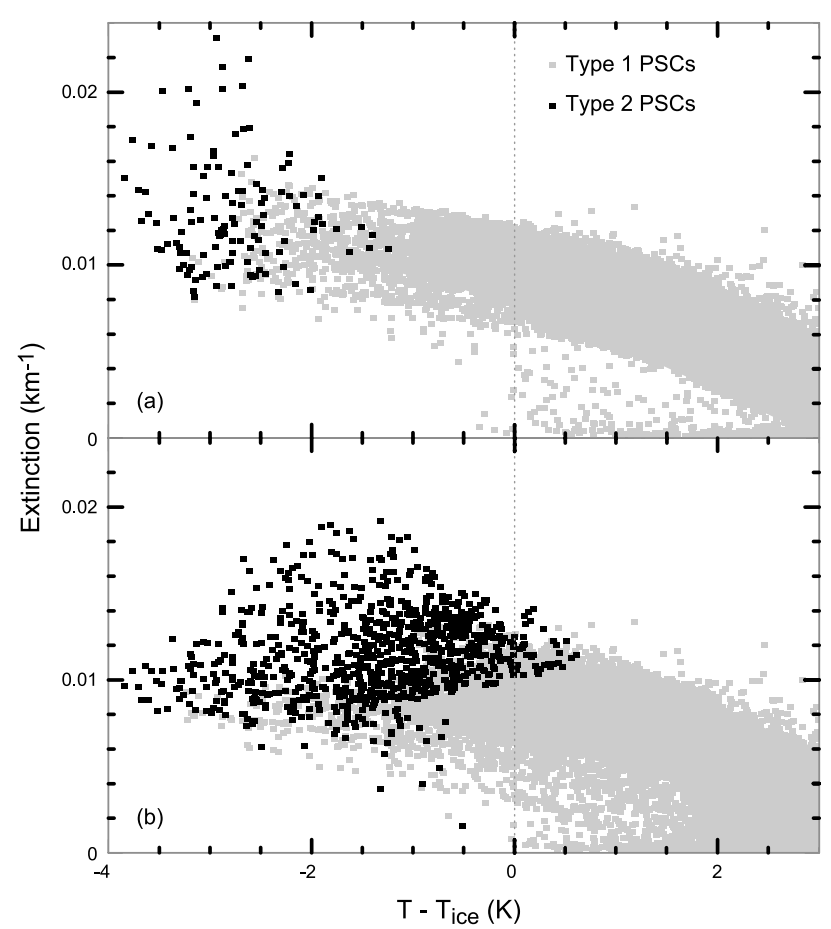

Fig. 7. Effect of the vapor deposition mechanism on PSC existence temperature. Results are plotted as PSC extinction at one micron vs. temperature. Temperature is relative to the ice frost point (dotted line) calculated assuming a constant 5 ppmv of water. Extinction is shown for all model points at which $\mathrm{T}^{-\mathrm{T}_{\text {ice }}}<3 \mathrm{~K}$. Type $2 \mathrm{PSCs}$ (defined as PSCs in which $>1 \%$ of the $\mathrm{H}_{2} \mathrm{O}$ is condensed as ice) are indicated by black points, while Type 1 PSCs are indicated by gray points. The top panel (a) shows results for a simulation with homogeneous ice nucleation alone. The bottom panel (b) shows results for a simulation in which ice nucleates on SAT, with $10^{-2} \mathrm{~cm}^{-3}$ SAT particles.

that the UKMO analysis was $1-2 \mathrm{~K}$ colder than other analyses during the coldest period of the winter (Drdla et al., 2003). Therefore, the model Type 2 PSCs cannot be readily compared with observations. However, the sensitivity of the model results to the ice formation process can be investigated.

Figure 7 demonstrates that adding ice nucleation on SAT to the simulations allows Type 2 PSCs (newly formed, fully developed, evaporating, etc.) to exist at warmer temperatures and, thus, occur more frequently. Results are plotted in terms of optical extinction, summed over all model particle types, to facilitate comparison with satellite measurements. Optical extinctions are calculated from the model size distributions using a Mie code (Steele et al., 1999). As Fig. 7a shows, if Type 2 PSCs can only form by homogeneous freezing (the same scenario as the "IceFrz" scenario in Drdla et al. (2003)), significant supercooling with respect to the ice frost point $(>2 \mathrm{~K})$ is required before Type 2 PSCs appear. Relatively few air parcels experience such cold temperatures, 
thereby reducing the number of PSC events. Furthermore, these PSCs tend to sublimate at cold temperatures since rapid dehydration lowers the frost point. (Determination of the ice frost point in Fig. 7 assumes a constant value of 5 ppmv $\mathrm{H}_{2} \mathrm{O}$ to mimic analyses applied to satellite data). On the other hand, nucleation on SAT permits Type 2 PSCs to exist at temperatures very near the ice frost point (Fig. 7b). The number of Type 2 PSC events is increased because more air parcels experience the required temperatures. Furthermore, homogeneous freezing of ice is suppressed when ice is able to nucleate on SAT. Even when temperatures fall several degrees below the ice frost point, the high supersaturations necessary for homogeneous freezing are prevented by water condensation onto the existing ice. Therefore, ice nucleation on SAT fundamentally changes the characteristics of the Type 2 PSCs at all temperatures. It is important to note that the Type 2 PSCs above the ice frost point in Fig. $7 \mathrm{~b}$ are real. Some are the result of finite evaporation timescales; a PSC is considered to be present until $99 \%$ of the $\mathrm{H}_{2} \mathrm{O}$ has evaporated. Others are in air that has been rehydrated. The additional water vapor allows Type 2 PSCs to persist at temperatures warmer than the 5 ppmv ice frost point shown in Fig. 7.

Similar features, namely more widespread Type 2 PSCs with events near the ice frost point, were found for a wide range of assumed SAT particle concentrations. However, the characteristics of the PSCs were strongly influenced by this assumption. Larger SAT particle concentrations allow larger ice particle concentrations, smaller particle radii, and larger extinctions. In turn, dehydration is affected, as shown in Fig. 8. Dehydration is four times more frequent, with more than $40 \%$ of the vortex experiencing some dehydration for all simulations with SAT concentrations of $10^{-3} \mathrm{~cm}^{-3}$ or higher (Fig. 8a). However, the dehydration associated with ice nucleation on SAT is less intense than for homogeneous freezing. Whereas homogeneous freezing causes dehydration that frequently exceeds $30 \%$ in individual air parcels, with nucleation on SAT almost all the air parcels experience less than $30 \%$ dehydration, and many experience less than $10 \%$ dehydration. This less intense dehydration can be attributed to two factors. First, with the vapor deposition mechanism, ice particles are able to form in less intense cooling events than is possible with homogeneous freezing. Consequently, the brief PSC duration limits the extent of dehydration. Second, for SAT concentrations greater than $10^{-2} \mathrm{~cm}^{-3}$, the large number of resulting ice particles are small in size, thereby reducing the efficiency of dehydration. As a result, vortexaveraged dehydration only increases by a factor of two to three (Fig. 8b). Dehydration is strongest for SAT concentrations near $10^{-2} \mathrm{~cm}^{-3}$, where the ice particle number and radius combine to yield the most efficient dehydration. Examination of the sources of the model Type 2 PSCs reveals that in all scenarios with SAT concentrations of $10^{-3} \mathrm{~cm}^{-3}$ or higher, homogeneous freezing to form ice is suppressed. However, for lower SAT concentrations ice supersaturations can exceed the homogeneous freezing threshold. For exam-

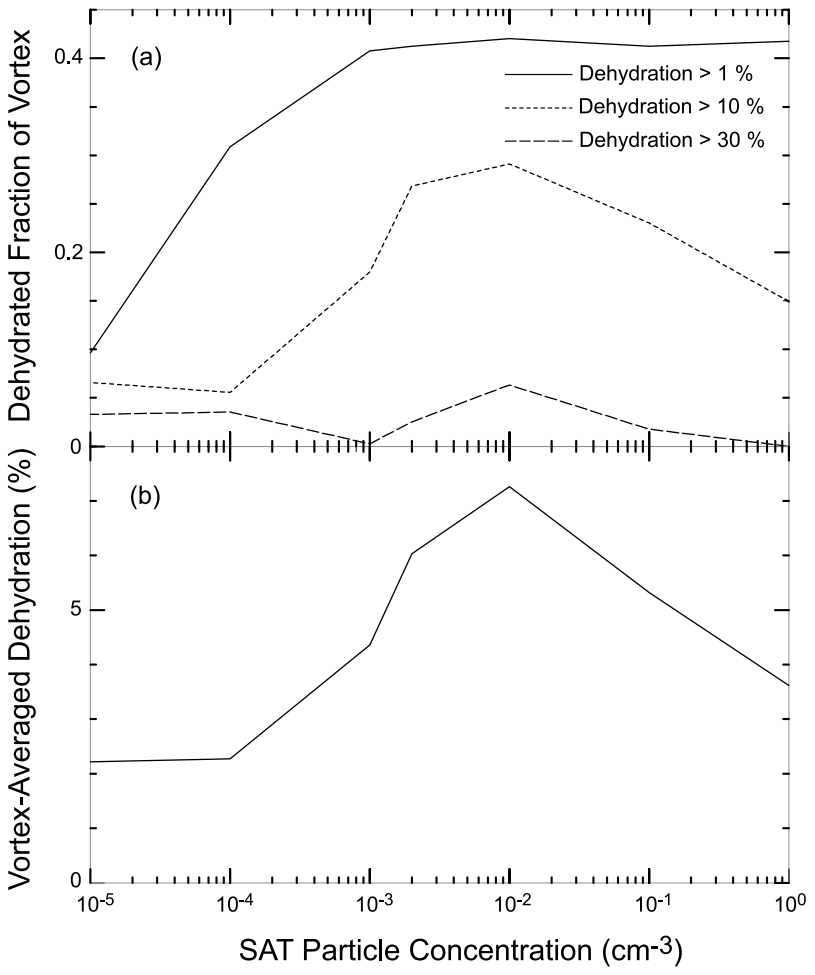

Fig. 8. Sensitivity of dehydration to assumed SAT particle concentration. Results are for $437.5-512.5 \mathrm{~K}$ potential temperature surfaces on 1 February 2000. At the lowest concentration shown $\left(10^{-5} \mathrm{~cm}^{-3}\right)$, the results are indistinguishable from a simulation in which no SAT particles are present. The top panel (a) shows the fraction of air parcels in the vortex experiencing any dehydration $(>1 \%)$, moderate dehydration $(>10 \%)$, and severe dehydration $(>30 \%)$. The bottom panel (b) shows the effect on the vortexaveraged dehydration.

ple, at a SAT concentration of $10^{-4} \mathrm{~cm}^{-3}$, only $25 \%$ of the model dehydration is a result of the vapor deposition of ice on SAT, while the rest is attributed to homogeneous freezing.

It is important to note that none of these simulations produced realistic denitrification or Type 1 PSC formation. A mechanism for directly forming NAT particles is necessary to reproduce these features. To confirm whether the results shown in Figs. 7 and 8 are applicable in more complete scenarios, an additional set of simulations was created. In these simulations, Type 1 PSCs were allowed to form by heterogeneous freezing of sulfate aerosols and ice nucleation was either turned off ( $m=0.75$ ) or turned on ( $m$ calculated using Eq. 7). Again, ice nucleation on SAT produced much more widespread Type 2 PSC formation and dehydration, and homogeneous freezing of ice was suppressed. Therefore, even in more realistic scenarios, SAT particles are present in sufficient concentrations for ice nucleation on SAT to control the characteristics of Type 2 PSCs.

Few observations exist that can be used to determine whether the model results are more realistic with or without 
ice nucleation on SAT, in part because relatively few studies have focused on Type 2 PSCs. However, POAM II satellite observations provide some information about the onset temperature of Type 2 PSCs (Fromm et al., 1999). Statistics of all Arctic POAM II Type 2 PSCs from 1993 to 1996 show an onset temperature near or above the ice frost point. These observations are qualitatively more consistent with the model results in which ice nucleation on SAT is considered (Fig. 7). Future work will exploit more detailed model/measurement comparisons to provide better tests of the Type 2 PSC formation mechanisms.

\section{Conclusions}

We have suggested an alternative formation mechanism for Type 2 PSCs, the vapor deposition of ice on SAT. Our laboratory results show that SAT is an efficient ice nucleus with $S_{\text {ice }}^{*}=1.3-1.02$ over the temperature range $169.8-194.5 \mathrm{~K}$. This corresponds to a necessary supercooling of $0.1-1.3 \mathrm{~K}$ below the ice frost point. Comparison of our results to previous work on the homogenous nucleation of ice out of liquid solution implies that this nucleation mechanism could be competitive, and perhaps even dominate, in Type 2 PSC formation. Furthermore, since the present work demonstrates that the vapor deposition of ice can occur at low $S_{\text {ice }}$, we recommend further study of the conditions required to deposit ice on NAT surfaces.

Using these experimental results in the IMPACT model allows us to probe the effect this heterogeneous nucleation mechanism could have on Type 2 PSC existence temperature, characteristics, and dehydration. The model simulations show ice nucleation on SAT permits Type 2 PSCs to form with little supercooling, thus increasing the extent of Type 2 PSCs relative to a simulation in which only homogeneous freezing is responsible for ice formation. As a result, the extent of dehydration is increased, although the severity of the dehydration is dependent upon the number of SAT nuclei assumed to be present.

Acknowledgement. This work was supported by grants from NASA-ACMAP (MDAR-0321-0132), NASA-AEAP, NASAUARP, and NSF (ATM-0137261). TJF was supported by an NSF Atmospheric Training Grant (EAR-9256339) and a CIRES Graduate Research Fellowship. The authors would like to thank R. Zhang and the anonymous referees for their helpful comments.

\section{References}

Anthony, S. E., Tisdale, R. T., Disselkamp, R. S., Tolbert, M. A., and Wilson, J. C.: FTIR studies of low temperature sulfuric acid aerosols, Geophys. Res. Lett., 22, 1105-1108, 1995.

Barone, S. B., Zondlo, M. A., and Tolbert, M. A.: A kinetic and product study of the hydrolysis of $\mathrm{ClONO}_{2}$ on Type 1a polar stratospheric cloud materials at $185 \mathrm{~K}$, J. Phys. Chem. A, 101, 8643-8652, 1997.
Bertram, A. K., Patterson, D. D., and Sloan, J. J.: Mechanisms and temperatures for the freezing of sulfuric acid aerosols measured by FTIR extinction spectroscopy, J. Phys. Chem., 100, 23762383, 1996.

Beyerle, G., Deckelmann, H., Neuber, R., Rosen, J. M., Reimer, E., and Schoeberl, M. R.: Occurrence of solid particles in the winter polar stratosphere above the nitric acid trihydrate coexistence temperature inferred from ground-based polarization lidar observations at Ny-Ålesund, Spitsbergen, J. Geophys. Res., 106, 2979-2992, 2001.

Browell, E. V., Butler, C. F., Ismail, S., Robinette, P. A., Carter, A. F., Higdon, N. S., Toon, O. B., Schoeberl, M. R., and Tuck, A. F.: Airborne lidar observations in the wintertime Arctic stratosphere: Polar stratospheric clouds, Geophys. Res. Lett., 17, 385388, 1990.

Carleton, K. L., Sonnenfroh, D. M., Rawlins, W. T., Wyslouzil, B. E., and Arnold, S.: Freezing behavior of single sulfuric acid aerosols suspended in a quadrupole trap, J. Geophys. Res., 102, 6025-6033, 1997.

Carslaw, K. S., Clegg, S. L., and Brimblecombe, P.: A thermodynamic model of the system $\mathrm{HCl}-\mathrm{HNO}_{3}-\mathrm{H}_{2} \mathrm{SO}_{4}-\mathrm{H}_{2} \mathrm{O}$, including solubilities of $\mathrm{HBr}$, from $<200 \mathrm{~K}$ to $328 \mathrm{~K}$, J. Phys. Chem., 99, 11 557-11 574,(http://mae.ucdavis.edu/ $\backslash$ char126wexler/aim/ papers.html), 1995.

Chang, H.-Y. A., Koop, T., Molina, L. T., and Molina, M. J.: Phase transitions in emulsified $\mathrm{HNO}_{3} / \mathrm{H}_{2} \mathrm{O}$ and $\mathrm{HNO}_{3} / \mathrm{H}_{2} \mathrm{SO}_{4} / \mathrm{H}_{2} \mathrm{O}$ solutions, J. Phys. Chem. A, 103, 2673-2679, 1999.

Chen, Y., DeMott, P. J., Kreidenweis, S. M., Rogers, D. C., and Sherman, D. E.: Ice formation by sulfate and sulfuric acid aerosol particles under upper-tropospheric conditions, J. Atmos. Sci., 57, 3752-3766, 2000.

Crutzen, P. J. and Arnold, F.: Nitric acid cloud formation in the cold Antarctic stratosphere: A major cause for the springtime "ozone hole", Nature, 324, 651-655, 1986.

David, C., Bekki, S., Godin, S., Mégie, G., and Chipperfield, M. P.: Polar stratospheric clouds climatology over Dumont d'Urville between 1989 and 1993 and the influence of volcanic aerosols on their formation, J. Geophys. Res., 103, 22 163-22 180, 1998.

DeMott, P. J., Rogers, D. C., and Kreidenweis, S. M.: The susceptibility of ice formation in upper tropospheric clouds to insoluble aerosol components, J. Geophys. Res., 102, 19575-19584, 1997.

Drdla, K.: Applications of a Model of Polar Stratospheric Clouds and Heterogeneous Chemistry, PhD thesis, University of California, Los Angeles, 1996.

Drdla, K., Schoeberl, M. R., and Browell, E. V.: Microphysical modeling of the 1999-2000 Arctic winter: 1. Polar stratospheric clouds, denitrification, and dehydration, J. Geophys. Res., 108, doi:10.1029/2001JD000782, 2003.

Drdla, K. and Turco, R. P.: Denitrification through PSC formation: A 1-D model incorporating temperature oscillations, J. Atmos. Chem., 12, 319-366, 1991.

Dye, J. E., Baumgardner, D., Gandrud, B. W., Kawa, S. R., Kelly, K. K., Loewenstein, M., Ferry, G. V., Chan, K. R., and Gary, B. L.: Particle size distributions in Arctic polar stratospheric clouds, growth and freezing of sulfuric acid droplets, and implications for cloud formation, J. Geophys. Res., 97, 8015-8034, 1992.

Eisenberg, D. and Kauzmann, W.: The Structure and Properties of Water, Oxford University Press, New York, 296, 1969. 
Fletcher, N. H.: Physics of Rainclouds, Cambridge University Press, London, 386, 1962.

Fortin, T. J., Shilling, J. E., and Tolbert, M. A.: Infrared spectroscopic study of the low-temperature phase behavior of ammonium sulfate, J. Geophys. Res., 107, doi:10.1029/2001JD000677, 2002.

Fromm, M. D., Bevilacqua, R. M., Hornstein, J., Shettle, E., Hoppel, K., and Lumpe, J. D.: An analysis of Polar Ozone and Aerosol Measurement (POAM) II Arctic polar stratospheric cloud observations, 1993-1996, J. Geophys. Res., 104, $24341-$ $24357,1999$.

Gable, C. M., Betz, H. F., and Maron, S. H.: Phase equilibria of the system sulfur trioxide-water, J. Am. Chem. Soc., 72, 1445-1448, 1950.

Gobbi, G. P. and Adriani, A.: Mechanisms of formation of stratospheric clouds observed during the Antarctic late winter of 1992, Geophys. Res. Lett., 20, 1427-1430, 1993.

Herman, R. L., Drdla, K., Spackman, J. R., Hurst, D. F., Popp, P. J., Webster, C. R., Romashkin, P. A., Elkins, J. W., Weinstock, E. M., Gandrud, B. W., Toon, G. C., Schoeberl, M. R., Jost, H., Atlas, E. L., and Bui, T. P.: Hydration, dehydration, and the total hydrogen budget of the 1999/2000 winter Arctic stratosphere, J. Geophys. Res., 108, doi:10.1029/2001JD001257, 2003.

Hobbs, P. V.: Ice Physics, Clarendon Press, Oxford, 837, 1974.

Iraci, L. T., Middlebrook, A. M., and Tolbert, M. A.: Laboratory studies of the formation of polar stratospheric clouds: Nitric acid condensation on thin sulfuric acid films, J. Geophys. Res., 100, 20 969-20 977, 1995.

Iraci, L. T., Fortin, T. J., and Tolbert, M. A.: Dissolution of sulfuric acid tetrahydrate at low temperatures and subsequent growth of nitric acid trihydrate, J. Geophys. Res., 103, 8491-8498, 1998.

Jensen, E. J. and Toon, O. B.: The potential impact of soot particles from aircraft exhaust on cirrus clouds, Geophys. Res. Lett., 24, 249-252, 1997.

Jensen, E. J., Toon, O. B., and Hamill, P.: Homogeneous freezing nucleation of stratospheric solution droplets, Geophys. Res. Lett., 18, 1857-1860, 1991.

Koop, T. and Carslaw, K. S.: Melting of $\mathrm{H}_{2} \mathrm{SO}_{4} \cdot 4 \mathrm{H}_{2} \mathrm{O}$ particles upon cooling: Implications for polar stratospheric clouds, Science, 272, 1638-1641, 1996.

Koop, T., Biermann, U. M., Raber, W., Luo, B. P., Crutzen, P. J., and Peter, Th.: Do stratospheric aerosol droplets freeze above the ice frost point?, Geophys. Res. Lett., 22, 917-920, 1995.

Koop, T., Carslaw, K. S., and Peter, Th.: Thermodynamic stability and phase transitions of PSC particles, Geophys. Res. Lett., 24, 2199-2202, 1997a.

Koop, T., Luo, B., Biermann, U. M., Crutzen, P. J., and Peter, Th.: Freezing of $\mathrm{HNO}_{3} / \mathrm{H}_{2} \mathrm{SO}_{4} / \mathrm{H}_{2} \mathrm{O}$ solutions at stratospheric temperatures: Nucleation statistics and experiments, J. Phys. Chem. A, 101, 1117-1133, 1997b.

Koop, T., Ng, H. P., Molina, L. T., and Molina, M. J.: A new optical technique to study aerosol phase transitions: The nucleation of ice from $\mathrm{H}_{2} \mathrm{SO}_{4}$ aerosols, J. Phys. Chem. A, 102, 8924-8931, 1998.

Larsen, N., Rosen, J. M., Kjome, N. T., and Knudsen, B.: Deliquescence and freezing of stratospheric aerosol observed by balloonborne backscattersondes, Geophys. Res. Lett., 22, 1233-1236, 1995.
Luo, B., Peter, Th., and Crutzen, P.: Freezing of stratospheric aerosol droplets, Geophys. Res. Lett., 21, 1447-1450, 1994.

Mancini, E., Pitari, G., and Visconti, G.: Dehydration in the Antarctic stratosphere: Radiative effects, Geophys. Res. Lett., 19, 585588, 1992.

Marti, J. and Mauersberger, K.: A survey and new measurements of ice vapor pressure at temperatures between 170 and $250 \mathrm{~K}$, Geophys. Res. Lett., 20, 363-366, 1993.

Massucci, M., Clegg, S. L., and Brimblecombe, P.: Equilibrium partial pressures, thermodynamic properties of aqueous and solid phases, and $\mathrm{Cl}_{2}$ production from aqueous $\mathrm{HCl}$ and $\mathrm{HNO}_{3}$ and their mixtures, J. Phys. Chem. A, 103, 4209-4226, (http://www. hpc1.uea.ac.uk/ char126e770/aim.html), 1999.

McElroy, M. B., Salawitch, R. J., Wofsy, S. C., and Logan, J. A.: Reductions of Antarctic ozone due to synergistic interactions of chlorine and bromine, Nature, 321, 759-762, 1986.

Middlebrook, A. M., Iraci, L. T., McNeill, L. S., Koehler, B. G., Wilson, M. A., Saastad, O. W., Tolbert, M. A., and Hanson, D. R.: Fourier transform-infrared studies of thin $\mathrm{H}_{2} \mathrm{SO}_{4} / \mathrm{H}_{2} \mathrm{O}$ films: Formation, water uptake, and solid-liquid phase changes, J. Geophys. Res., 98, 20 473-20 481, 1993.

Molina, M. J.: Heterogeneous chemistry on polar stratospheric clouds, Atmos. Environ., 25A, 2535-2537, 1991.

Molina, M. J., Zhang, R., Wooldridge, P. J., McMahon, J. R., Kim, J. E., Chang, H.-Y. A., and Beyer, K. D.: Physical chemistry of the $\mathrm{H}_{2} \mathrm{SO}_{4} / \mathrm{HNO}_{3} / \mathrm{H}_{2} \mathrm{O}$ system: Implications for polar stratospheric clouds, Science, 261, 1418-1423, 1993.

Nagai, T., Uchino, O., Itabe, T., Shibata, T., Mizutani, K., and Fujimoto, T.: Polar stratospheric clouds observed at Eureka $\left(80^{\circ} \mathrm{N}\right.$, $86^{\circ} \mathrm{W}$ ) in the Canadian Arctic during the 1994/1995 winter, Geophys. Res. Lett., 24, 2243-2246, 1997.

Nash, K. L., Sully, K. J., and Horn, A. B.: Infrared spectroscopic studies of the low temperature interconversion of sulfuric acid hydrates, Phys. Chem. Chem. Phys., 2, 4933-4940, 2000.

Peter, Th.: Microphysics and heterogeneous chemistry of polar stratospheric clouds, Annu. Rev. Phys. Chem., 48, 785-822, 1997.

Pruppacher, H. R. and Klett, J. D.: Microphysics of Clouds and Precipitation, Kluwer Academic Publishers, Norwell, MA, 954, 1997.

Rivière, E. D., Huret, N., Taupin, F. G., Renard, J.-B., Pirre, M., Eckermann, S. D., Larsen, N., Deshler, T., Lefèvre, F., Payan, S., and Camy-Peyret, C.: Role of lee waves in the formation of solid polar stratospheric clouds: Case studies from February 1997, J. Geophys. Res., 105, 6845-6853, 2000.

Rosen, J. M., Kjome, N. T., and Oltmans, S. J.: Simultaneous ozone and polar stratospheric cloud observations at South Pole Station during winter and spring 1991, J. Geophys. Res., 98, 12741$12751,1993$.

Sassen, K. and Benson, S.: Ice nucleation in cirrus clouds: A model study of the homogeneous and heterogeneous modes, Geophys. Res. Lett., 27, 521-524, 2000.

Sassen, K., Peter, Th., Luo, B. P., and Crutzen, P. J.: Volcanic Bishop's ring: Evidence for sulfuric acid tetrahydrate particle aureole, Appl. Optics, 33, 4602-4606, 1994.

Schiller, C., Engel, A., Schmidt, U., Borchers, R., and Ovarlez, J.: The partitioning of hydrogen species in the Arctic winter stratosphere: Implications for microphysical parameters, J. Geophys. Res., 101, 14 489-14 493, 1996. 
Schiller, C., Bauer, R., Cairo, F., Deshler, T., Dörnbrack, A., Elkins, J., Engel, A., Flentje, H., Larsen, N., Levin, I., Müller, M., Oltmans, S., Ovarlez, H., Ovarlez, J., Schreiner, J., Stroh, F., Voigt, C., and Vömel, H.: Dehydration in the Arctic stratosphere during the SOLVE/THESEO-2000 campaigns, J. Geophys. Res., 107, doi:10.1029/2001JD000463, 2002.

Solomon, S., Garcia, R. R., Rowland, F. S., and Wuebbles, D. J.: On the depletion of Antarctic ozone, Nature, 321, 755-758, 1986.

Stanford, J. L. and Davis, J. S.: A century of stratospheric cloud reports: 1870-1972, Bull. Am. Meteorol. Soc., 55, 213-219, 1974.

Steele, H. M., Drdla, K., Turco, R. P., Lumpe, J. D., and Bevilacqua, R. M.: Tracking polar stratospheric cloud development with POAM II and a microphysical model, Geophys. Res. Lett., 26, 287-290, 1999.

Tabazadeh, A., Jensen, E. J., and Toon, O. B.: A model description for cirrus cloud nucleation from homogeneous freezing of sulfate aerosols, J. Geophys. Res., 102, 23 845-23 850, 1997 a.

Tabazadeh, A., Toon, O. B., and Jensen, E. J.: Formation and implications of ice particle nucleation in the stratosphere, Geophys. Res. Lett., 24, 2007-2010, 1997b.
Tabazadeh, A., Santee, M. L., Danilin, M. Y., Pumphrey, H. C., Newman, P. A., Hamill, P. J., and Mergenthaler, J. L.: Quantifying denitrification and its effect on ozone recovery, Science, 288, 1407-1411, 2000.

Toon, O. B., Hamill, P., Turco, R. P., and Pinto, J.: Condensation of $\mathrm{HNO}_{3}$ and $\mathrm{HCl}$ in the winter polar stratospheres, Geophys. Res. Lett., 13, 1284-1287, 1986.

Turco, R. P., Toon, O. B., and Hamill, P.: Heterogeneous physicochemistry of the polar ozone hole, J. Geophys. Res., 94, 16493 16510, 1989.

Zhang, R., Wooldridge, P. J., Abbatt, J. P. D., and Molina, M. J.: Physical chemistry of the $\mathrm{H}_{2} \mathrm{SO}_{4} / \mathrm{H}_{2} \mathrm{O}$ binary system at low temperatures: Stratospheric implications, J. Phys. Chem., 97, 73517358, 1993.

Zhang, R., Leu, M.-T., Molina, M.J.: Formation of polar stratospheric clouds on preactivated background aerosols, Geophys. Res. Lett., 23, 1669-1672, 1996.

Zuberi, B., Bertram, A. K., Koop, T., Molina, L. T., and Molina, M. J.: Heterogeneous freezing of aqueous particles induced by crystallized $\left(\mathrm{NH}_{4}\right)_{2} \mathrm{SO}_{4}$, ice and letovicite, J. Phys. Chem. A, 105, 6458-6464, 2001. 